\title{
A Steady-Hand Robotic System for Microsurgical Augmentation
}

\author{
Russell Taylor ${ }^{1}$, Patrick Jensen ${ }^{3}$, Louis Whitcomb ${ }^{2}$, Aaron Barnes ${ }^{2}$, Rajesh Kumar ${ }^{1}$, \\ Dan Stoianovici ${ }^{4}$, Puneet Gupta ${ }^{3}$, ZhengXian Wang ${ }^{1}$, \\ Eugene deJuan ${ }^{3}$, and Louis Kavoussi ${ }^{4 *}$
}

\author{
${ }^{1}$ Department of Computer Science, Whiting School of Engineering \\ ${ }^{1}$ Department of Mechanical Engineering, Whiting School of Engineering \\ ${ }^{3}$ Wilmer Eye Institute, Johns Hopkins Medical Institutions \\ ${ }^{4}$ James Buchanan Brady Urological Institute, Johns Hopkins Medical Institutions \\ The Johns Hopkins University, Baltimore, Maryland, USA
}

\begin{abstract}
This paper reports the development of a robotic system designed to extend a human's ability to perform small-scale (sub-millimeter) manipulation tasks requiring human judgement, sensory integration and hand-eye coordination. Our novel approach, which we call "steady hand" micromanipulation, is for tools to be held simultaneously both by the operator's hand and a specially designed actively controlled robot arm. The robot's controller senses forces exerted by the operator on the tool and by the tool on the environment, and uses this information in various control modes to provide smooth, tremor-free precise positional control and force scaling. Our goal is to develop a manipulation system with the precision and sensitivity of a machine, but with the manipulative transparency and immediacy of handheld tools for tasks characterized by compliant or semi-rigid contacts with the environment.
\end{abstract}

\section{Introduction}

This paper describes the first steps in an ongoing development of a robotic assistant for microsurgery and other precise manipulation tasks. It reports a new robotic system developed to extend a human's ability to perform small-scale (sub-millimeter) manipulation tasks requiring human judgement, sensory integration and hand-eye coordination. Our approach, which we call "steady hand" micro-manipulation is for tools to be held simultaneously both by the operator's hand and a specially designed robot arm (figure 1). The robot's controller senses forces exerted by the operator on the tool and by the tool on the environment, and uses this information in various control modes to provide smooth, tremor-free precise positional control and force scaling. The result will be a manipulation system with the precision and sensitivity of

We gratefully acknowledge the support of the National Science Foundation under grant \#IIS9801684, the Engineering Research Center grant \#EEC9731478, and in cooperation with the Whitaker Foundation on grant \#ST32HL07712. General lab infrastructure was partially supported by NSF equipment grants CDA-9529509 and EIA9703080 and by an equipment grant from Intel. This work was also funded in part by Johns Hopkins internal funds. 
a machine, but with the manipulative transparency and immediacy of hand-held tools for tasks characterized by compliant or semi-rigid contacts with the environment.

Humans possess superb manual dexterity, visual perception, and other sensorymotor capabilities. We manipulate best at a "human scale" dictated by our physical size and manipulation capabilities and roughly corresponding to the tasks routinely performed by our cave man ancestors. Tasks that require very precise, controlled motions are difficult or impossible for most people. Further, humans work best in tasks that require relative positioning or alignment based on visual or tactile feedback. We do not come equipped with an innate ability to position or fabricate objects accurately relative to arbitrary measuring standards or to perform tasks based on nonhuman sensory feedback. For these tasks, we rely on machines. A good machine tool, for example, can routinely measure and fabricate parts to a precision of $\approx 2.5 \mu \mathrm{m}$ $(\approx 0.001$ inch). Fine-scale tasks such as microsurgery require both precise manipulation and human judgement. Other tasks may require combining precise manipulation with sources of information (assembly specifications, non-visible-light images, etc.) that are not naturally available to a human. We thus have a choice: either automate the human judgement aspects of the task (difficult at best and often impossible) so that a machine can automatically perform the task or else find a way to use a machine to augment human manipulation capabilities while still exploiting the human's natural strengths.

Most prior robotic micro-manipulation systems have emphasized traditional master-slave and telerobotic manipulation. Our approach might offer several advantages compared to these systems in the context of micromanipulation. These include: 1) simplicity; 2) potentially cheaper implementations; 3) a more direct coupling to the human's natural kinesthetic senses; 4) straightforward integration into existing application environment; and 5) greater "immediacy" for the human operator. The principal drawbacks are the loss of the ability to "scale" positional motions and the loss of the ability to manipulate objects remotely. These are certainly important abilities, but we believe there are many tasks in which they are not crucial and for which a simpler alternative is more attractive. These advantages are especially attractive in applications like microsurgery, where surgeon acceptance is crucial and where approaches that do not require a complete re-engineering of the surgical workstation are much easier to introduce into practice.

\section{Robotically Assisted Micro-Manipulation}

Mechanical systems have been developed which extend the capability of human operators using telerobotic principles [1] including virtual training [2], manipulation of objects in hazardous environments [3], remote surgery [4, 5], and microsurgery [2,6-10]. In general, telerobotic devices rely on an operator commanding the motion of a robot using a secondary input device. The operator may reside in close proximity to the robot, observing its motions through a microscope as in microsurgery or may be many miles away as in space exploration. In both cases, the operator is an integral part of the system and has direct control over how the manipulator moves. An ideal teleoperated system would be transparent to the operator and give the impression of direct control. The input device manipulated by the operator may be either passive, such as a trackball, joystick or stylus, or made up of active devices 
such as motors. An active input device allows forces imposed on the robot to be measured, scaled and mimicked at the input device to be subsequently felt by the operator.

Several systems have been developed for teleoperated microsurgery using a passive input device for operator control. Guerrouad and Vidal [11] describe a system designed for ocular vitrectomy in which a mechanical manipulator was constructed of curved tracks to maintain a fixed center of rotation. A similar micromanipulator [12] was used for acquiring physiological measurements in the eye using an electrode. While rigid mechanical constraints were suitable for the particular applications in which they were used, the design is not flexible enough for general purpose microsurgery and the tracks take up a great deal of space around the head. An ophthalmic surgery manipulator built by Jensen et al. [8] was designed for retinal vascular microsurgery and was capable of positioning instruments at the surface of the retina with sub-micron precision. While a useful experimental device, this system did not have sufficient range of motion to be useful for general-purpose microsurgery. Also, the lack of force sensing prevented the investigation of force/haptic interfaces in the performance of microsurgical tasks.

Many microsurgical devices $[2,6,7,9,10]$ are based on force-reflecting masterslave configurations. This paradigm allows an operator to grasp the master manipulator and apply forces. Forces measured on the master are scaled and reproduced at the slave and, if unobstructed, will cause the slave to move accordingly. Likewise, forces encountered by the slave are scaled and reflected back to the master. This configuration gallows position commands from the master to result in a reduced motion of the slave and for forces encountered by the slave to be amplified at the master. While a force-reflecting master-slave microsurgical system provides the surgeon with increased precision and enhanced proprioception, there are some drawbacks to such a design. The primary disadvantage is with complexity and cost since two mechanical systems, one for the master and one for the slave, are required. Another problem with telesurgery in general is that the surgeon is not allowed to directly manipulate the instrument used for the microsurgical procedure. While physical separation is necessary for systems designed to perform remote sutgery, it is not required during microsurgical procedures. In fact, surgeons are more likely to accept assistive devices if they are still allowed to directly manipulate the instruments.

\subsection{Shared Autonomy and Cooperative Control}

There is a large body of literature concerning provably stable control techniques for robots. Standard paradigms include 1) pre-programmed trajectory control of position [13,14] and force [15,16]; 2) fully autonomous robots (e.g. [17-19]); and 3) master-slave teleoperators (e.g., [20-22]). In our case, we are interested in developing provably stable controls for cases where both the robot and the human manipulate a single tool in contact with a compliant environment. The work most relevant to this includes that of Kazarooni [23-25] who developed exoskeletons to amplify the strength of a human operator.

Kazarooni et al. [23-25] report a linear systems analysis of the stability and robustness of cooperative human-robot manipulator control systems in which the manipulator scales-up the human operator's force input by a factor of $\approx 10$. The authors report a stability analysis of this closed-loop system (comprising a dynamical 
model of both the robot arm and the human arm) is complicated by the fact that precise mathematical plant models do not exist for either the hydraulically actuated robot and the operator's human arm. In consequence, in [23-25] the authors perform a robustness analysis to develop stable robot force-control laws that accommodate wide variation in both human and robot arm dynamics. In contrast, we propose to address the control problem of cooperative human-robot manipulator systems in which the manipulator scales-down the human operator's force input by a factor of $\approx 0.1$. To achieve this scaling-down of human input we anticipate comparable (or greater) difficulties to arise from unknown human arm dynamics, we can construct the system using electrical motors (rather than hydraulic motors) for which accurate dynamical models are available. A number of authors (e.g., [22,26]) have investigated "shared autonomy" and for cooperative control of teleoperators, typically with space or other "remote" applications where time delays can affect task performance. There has also been some work (e.g., [27]) on control of robots working cooperatively with humans to carry loads and do other gross motor tasks relevant for construction and similar applications. Within the area of surgery, we have long used "hands on" guiding of robots for positioning within the operating room (e.g., in the "Robodoc" [28-30] hip replacement surgery system and in the JHU/IBM LARS system [31-38] for endoscopic surgery). Davies et. al.[39-41], have combined handson guiding with position limits and have demonstrated 3 DOF machining of shapes in the end of a human tibia.

At JHU, we have been using the LARS robot [31] to perform a variety of "steady hand" tasks combining hand guiding, active control, and safety constraints in neuroendoscopy and other areas. In one experiment using the LARS robot-assisted evacuation of simulated hematomas was found to take longer (6.0 min vs $4.6 \mathrm{~min})$ than freehand evacuation but was found to remove much less unintended material $(1.5 \%$ vs $15 \%)$ [36]. We have also made some preliminary experiments using the LARS for micro-manipulation [42], although the compliance of the LARS upper linkage severely limits the benefit gained.

\section{A Robotic System for Steady-Hand Micro-Manipulation}

\subsection{Design Goals}

Table 1 summarizes the performance design goals for our system. Cooperative micro-manipulation requires capabilities not commonly found in conventional robots or teleoperator systems. Typically, these tasks will be performed by a human operator looking through a microscope while grasping a "handle" on the instrument or tool being used to perform the task. In the tasks that we are considering, we believe that motion "scaling" (in the sense that a $1 \mathrm{~cm}$ human hand motion might cause a $100 \mu \mathrm{m}$ instrument motion) is much less important than smooth motion naturally aligned with the human's own kinesthetic senses. Pulling on the tool's handle should produce intuitively natural translation and orientation motions.

We are interested in manipulation tasks requiring very precise positional control, with controlled end-effector motion resolution on the order of 3-10 $\mu \mathrm{m}$, when rotational motion is decoupled at the tool tip and 5-25 $\mu \mathrm{m}$ tip resolution when motion is decoupled about a fulcrum point $2 \mathrm{~cm}$ from the tool tip (i.e., when a point $2 \mathrm{~cm}$ 


\begin{tabular}{|c|l|}
\hline Base (XYZ) assembly & (Off-the-shelf) \\
\hline Work volume & $100 \mathrm{~mm} \times 100 \mathrm{~mm} \times 100 \mathrm{~mm}$ \\
\hline Top Speed & $40 \mathrm{~mm} / \mathrm{sec}$ \\
\hline Positioning resolution & $\approx 2.5 \mu \mathrm{m}(0.5 \mu \mathrm{m}$ encoder res.) \\
\hline RCM orientation assembly & (Custom) \\
\hline Link length & $100 \mathrm{~mm}$ \\
\hline Range of motion & Continuous $360^{\circ}$ \\
\hline Top speed & $180^{\circ} / \mathrm{sec}$ \\
\hline Angular resolution & $\approx 0.05^{\circ}\left(0.01^{\circ}\right.$ encoder res.) \\
\hline End-of-arm/guiding assembly & $($ Custom) \\
\hline Range of motion & $\pm 2 \mathrm{~cm} ; 360^{\circ}$ continuous \\
\hline Positioning resolution & $0.5 \mu \mathrm{m} ; 0.1^{\circ}$ (tentative) \\
\hline Top speed & $40 \mathrm{~mm} / \mathrm{sec} ; 180^{\circ} / \mathrm{sec}$ \\
\hline
\end{tabular}

Table 1. Steady-Hand Robot Design Goals

from the tool tip remains fixed in space). Our strong preference is for relatively low power actuators with high-reduction, non-backdrivable joints. Such systems are relatively easy to monitor, stop, and stay put once stopped. For a clinical system, we also require some form of redundant position sensing. Although the current implementation (intended for pre-clinical research) does not have this feature, future implementations will have this feature.

We are primarily interested in manipulation tasks with a reasonable degree of contact compliance between the tool and the environment being manipulated. In the case of microsurgery, this compliance is provided by the tissue being manipulated. Our goal is moderate bandwidth $(3-5 \mathrm{~Hz})$ control and scaling of interaction forces, with tool tip forces ranging from $\approx 0.001 \mathrm{~N}$ to $\approx 0.01 \mathrm{~N}$, depending on specific application, and human interaction forces ranging from $\approx 0.03 \mathrm{~N}$ to $\approx 3 \mathrm{~N}$. We also wish to provide higher bandwidth sensing and haptic feedback of force discontinuities, and to explore the usefulness of such feedback in micro-manipulation tasks. One option under consideration is the addition of vibrotactile displays [43]

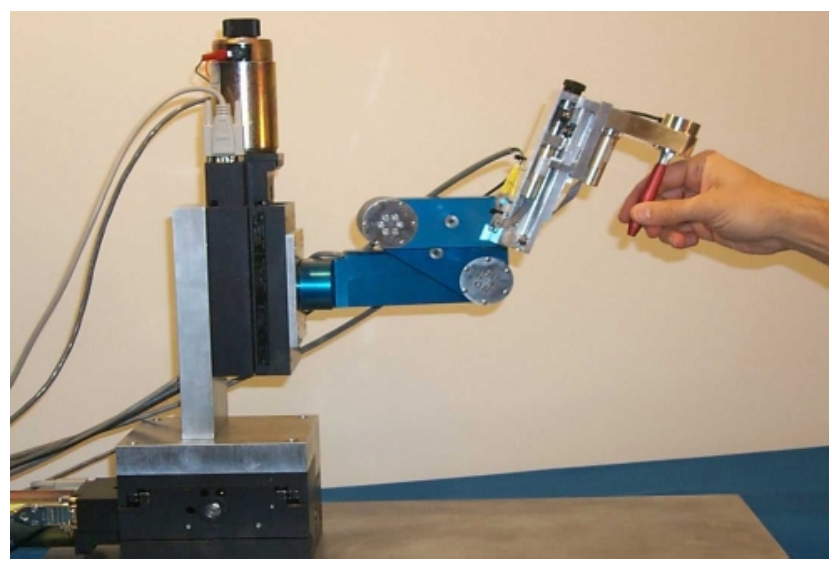

Figure 1. Current version of the steady hand manipulator 

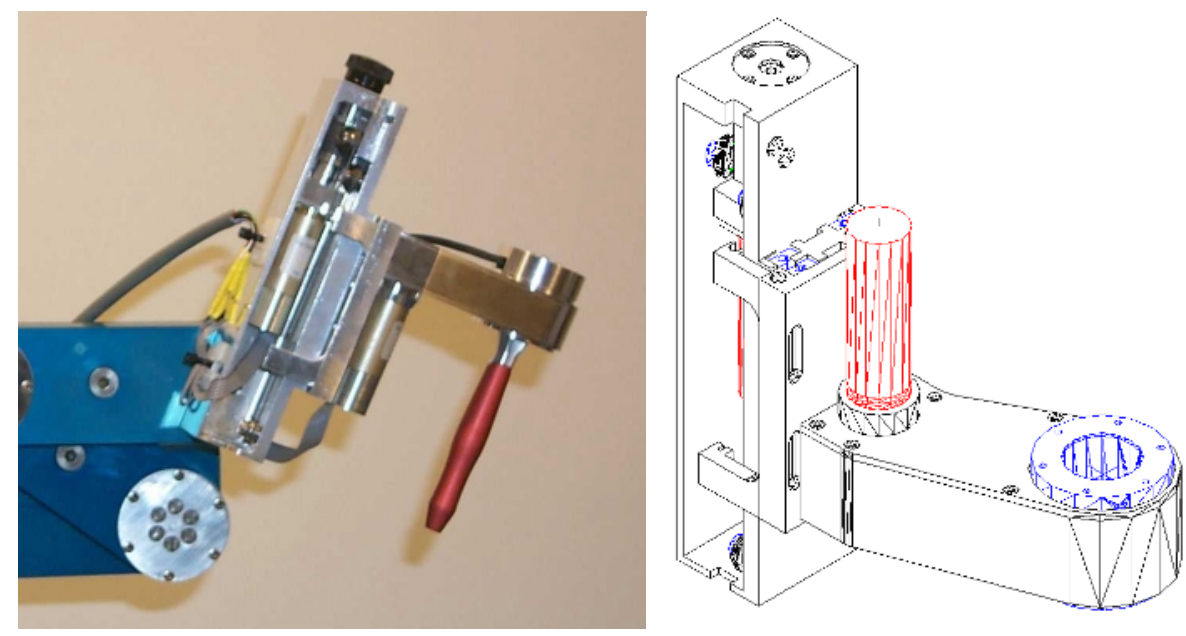

Figure 2. Photograph (left) and drawing (right) of insertion and rotation stages

\subsection{System Design and Implementation}

\subsubsection{Manipulator and Mechanical Components}

We have emphasized modularity in development of our steady hand system. The manipulator itself (Figure 1) kinematically decouples surgical instrument translational and rotational motions and consists of four modular components. The XYZ translation assembly is constructed with off-the-shelf motorized micrometer stages from New England Affiliated Technologies of Lawrence, MA.. Each axis has $100 \mathrm{~mm}$ of travel, can travel at speeds $>40 \mathrm{~mm} / \mathrm{sec}$ and has a positioning resolution of $<2.5 \mu \mathrm{m}(0.5 \mu \mathrm{m}$ encoder resolution).

The remote center-of-motion (RCM) orientation assembly provides two rotations about a "fulcrum" or remote motion center point located in free space approximately $100 \mathrm{~mm}$ from the robot. The current compact design [44,45] weighs $1.6 \mathrm{Kg}$ and may be folded into a $171 \times 69 \times 52 \mathrm{~mm}$ cube. The RCM design is very well adapted to microsurgical augmentation, since it permits us optimize actuators to combine relatively rapid reorientations about a fixed point with very precise and relatively slow translational motions. Its kinematic properties make it appropriate for a number of endoscopic and percutaneous applications, and it has been used clinically at JHU for placing needles into the filling system of the kidney under fluoroscopic guidance [46,47].

The instument insertion stage provides linear displacement along the tool axis passing through the remote motion center. The axis utilizes a two-stage telescoping crossed-roller slide mechanism driven via a cable by an encoded DC servo motor. The insertion stage can travel at speeds of $\approx 30 \mathrm{~mm} / \mathrm{sec}$ and has a positioning resolution of $\approx 5-10 \mu \mathrm{m}$ (1.5 $\mu \mathrm{m}$ encoder resolution). The rotation end-effector provides rotation about the tool axis and the mounting surface for the force sensor with guiding handle. The instrument rotation stage is driven by a timing belt attached to a encoded DC 
servo motor. It provides a $360^{\circ}$ continuous range of motion and is expected to travel at speeds of $\approx 120^{\circ}-180^{\circ} / \mathrm{sec}$ with a positioning resolution of $\approx 0.05^{\circ}-0.10^{\circ}$ ( $0.01^{\circ}$ encoder resolution).

The current force sensing handle uses a commercially available 6DOF force sensor (Model: NANO-17 SI 12/0.12, ATI Industrial Automation, NC) to capture user forces. The force sensor is read using a 12-bit ISA bus F/T controller card with up to $7800 \mathrm{~Hz}$ sampling rates, and has $0.025 \mathrm{~N}$ resolution in $\mathrm{Z}$ and $0.0625 \mathrm{~N}$ in $\mathrm{XY}$. The force sensor is mounted on the instrument rotation stage with its $\mathrm{z}$-axis parallel to the instrument insertion stage of the robot.

A variety of surgical instruments such as picks, forceps, needle holders and scissors are required during microsurgical procedures. To utilize the benefits offered by the cooperative control algorithms of steady hand augmentation, these microsurgical tools must be equipped with sensitive, multidimensional force sensors. Our initial approach uses silicon strain gauges configured into bridges located within the surgical tool handle.

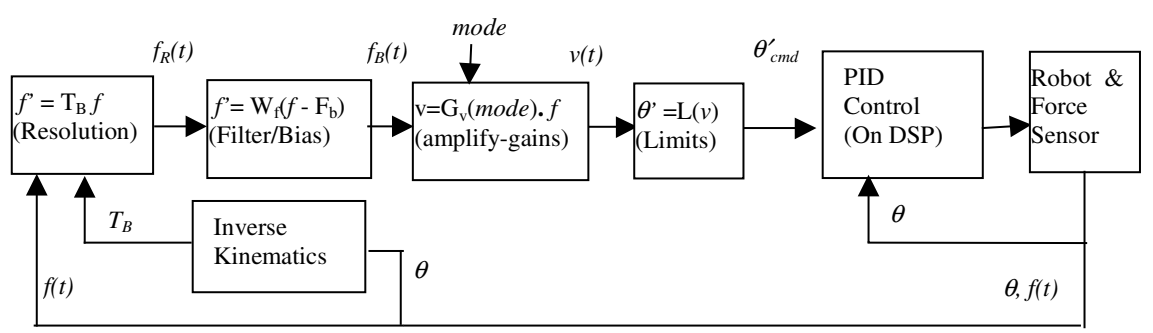

Figure 3. Controller block diagram. The notation is as follows. $f(t)$ : sampled forces $\left(f_{x}, f_{y}, f_{z}\right) ; f_{R}(t)$ : forces resolved in the robot base frame, $f_{B}(t)$ : filtered and biased forces; $F_{b}$ :bias force forces $\left(f_{b x}, f_{b y}, f_{b z}\right)$, in the robot base frame; $\mathrm{T}_{B}$ : transformation from force sensor frame to robot base frame; mode: Base $\mathrm{X}, \mathrm{Y}$ joints and insertion joint (mode 1), or RCM rotation joints and insertion joint (mode 2$) ; \mathrm{G}_{\mathrm{v}}($ mode): joint velocity proportional gains, based on user selected mode; $v(t)$ : joint velocities for selected joints; $\theta$ : joint position feedback from encoders; $\theta_{\text {cmd }}$ :commanded joint velocities.

\subsubsection{Control System}

The robot hardware control runs on a Pentium-II 450MHz PC with the Windows NT operating system. An 8-axis DSP series controller card (PCX/DSP, Motion Engineering Inc, CA) is used to control the robot. The card provides servo control using a 40MHZ Analog Devices ADSP-2105 processor. It also has support for user digital and analog input, output lines. The PC also houses the ISA force sensor controller.

Although we anticipate that the control algorithms will eventually be quite sophisticated, the current method (illustrated in Figure 3) is very simple. User forces are sensed from the force sensor attached axially to the handle held by the user. The $\mathrm{z}$ axis of the force sensor is aligned with the axis of insertion of the robot and the other 
two axis are aligned when the instrument rotation stage is at zero. The force sensor is calibrated by computing a bias force with no external forces on the sensor. This bias force is stored in the robot base coordinate frame. During compliant motion, the system samples the current forces, and resolves them to the robot base frame. These resolved forces are offset by the bias and noise filtered using a simple filter $\left(\mathrm{W}_{\mathrm{f}}\right)$. This biased signal is then amplified by gains $G_{v}$. The velocities computed are limited by user specified limits. The commanded velocity is then supplied to the PID controller on the DSP.

\section{Current Status and Future Evolution}

Mechanical integration of the steady hand manipulation system is complete Experiments designed to evaluate the augmentation value of steady-hand manipulation are currently being performed. Initial indications are that the basic design assumptions of a stiff robot with force control are valid for surgical manipulations at a micro-scale. In one experimental study comparing unassisted human versus steady hand performance in inserting a 10-0 surgical needle into holes of diameter ranging from $150 \mu \mathrm{m}$ to $250 \mu \mathrm{m}$, the steady hand system improved success rates from $43 \%$ unassisted to $79 \%$ for $150 \mu \mathrm{m}$ holes and from $49 \%$ unassisted to $78 \%$ for $250 \mu \mathrm{m}$ holes [48].

Our immediate goal is a rigorous evaluation of the completed system as a microsurgery augmentation aid, using test environments developed by our colleagues at JHU's Wilmer Eye Institute and CMU [49-52]. We will compare the system invitro and in cadaveric models both against unassisted humans and against alternative methods for reducing physiological tremor (e.g., [50,53]). Subsequently, we hope to begin evaluation of a clinical system. Initial targeted applications will include epiretinal surgery and retinal vein cannulation under direct surgeon control.

A second stage will combine the steady hand system with various real time imaging modalities (video from optical microscopes \& endoscopes (e.g., [54], optical coherence tomography, etc.) in order to produce an enhanced mosaic image of the patient's eye. This information will be made available to the surgeon, for example, by image injection into the surgical microscope or by a suitable video display.

This "information enhanced" surgery system will gradually evolve into a more capable surgical assistant. Initial tasks will be rather simple. We anticipate the development of graceful ways to hand off control between the surgeon and the robot for the performance of specific surgical macros. For example, the surgeon may guide an injection instrument to the vicinity of a blocked vein, but rely on a specialized function incorporating visual servoing and force sensing to perform cannulation and injection of clot-dissolving drugs. Other examples include such "third hand" tasks as pointing a micro-endoscope at designated anatomical features or following the surgeon's instrument movements, force-controlled retraction, or the like. As this repertoire of functions increases, the system will become an increasingly effective partner in surgical treatment. 


\section{Summary}

Our approach extends earlier work on cooperative manipulation to microsurgery and focuses on performance augmentation utilizing both force and position control. Our goal is to develop a manipulation system with the precision and sensitivity of a machine, but with the manipulative transparency and immediacy of hand-held tools for tasks characterized by compliant or semi-rigid contacts with the environment. The design is highly modular and represents one step in the evolution of a family of robotic surgical devices. Although our first focus is retinal microsurgery, we believe that our approach is more general. Other applications will include neuroendoscopy, ENT, and microvascular surgery.

\section{References}

1. Sheridan, T.B., Teleoperation, telerobotics and telepresence: a progress report. Control Engineering Practice, 1995. 3(2): p. 205-214.

2. Hunter, I.W., et al., Ophthalmic microsurgical robot and associated virtual environment. Computers in Biology and Medicine, 1995. 25(2): p. 173-182.

3. Mindell, D.A., et al. JasonTalk: A standard ROV vehicle control system. in IEEE/MTS OCEANS'93. 1993.

4. Satava, r., Robotics, telepresence, and virtual reality: A critical analysis fo the future of surgery. Minimally Invasive Therapy, 1992. 1: p. 357-363.

5. Green, P., et al., Telepresence: Advanced Teleoperator Technology ofr Minimally Invasive Surgery (abstract). Surgical Endoscopy, 1992. 6(91).

6. Charles, S., Dexterity enhancement for surgery. Proc First Int'l Symp Medical Robotics and Computer Assisted Surgery, 1994. 2: p. 145-160.

7. Misuishi, M., et al. Dexterity enhancement for a tele-micro-surgery system with multiple macro-micro co-located operation point manipulators and understanding of the operator's intention. in First joint conference computer vision, virtual realtiy and robotics in medicine and medical robotics and computer-assisted surgery. 1997. Grenoble, France: Springer.

8. Jensen, P.S., et al., Toward robot assisted vascular microsurgery in the retina. Graefes Arch Clin Exp Ophthalmol, 1997. 235(11): p. 696-701.

9. Salcudean, S.E., S. Ku, and G. Bell. Performance measurement in scaled teleoperation for microsurgery. in First joint conference computer vision, virtual realtiy and robotics in medicine and medical robotics and computer-assisted surgery. 1997. Grenoble, France: Springer.

10. Schenker, P.S., H.O. Das, and R. Timothy. Development of a new high-dexterity manipulator for robot-assisted microsurgery. in Proceedings of SPIE - The International Society for Optical Engineering: Telemanipulator and Telepresence Technologies. 1995. Boston, MA.

11. Guerrouad, A. and P. Vidal, S.M.O.S.: Stereotaxical Microtelemanipulator for Ocular Surgery. Proc. of the Annual Int'l Conf. of the IEEE Engineering in Medicine and Biology Society, 1989: p. 11:879-880.

12. Pournaras, C.J., et al., New ocular micromanipulator for measurements of retinal and vitreous physiologic parameters in the mammalian eye. Exp Eye Res, 1991. 52: p. 723727.

13. Dinsmoor, C. and P. Hagermann. Fanuc robotics system r-j controller. in Proceedings of International Robots and Vision Automation Conference. 1993. Detroit Michigan USA.

14. Sakakibara, S. A two-armed intelligent robot assembles mini robots automatically. in Proceedings of the 1996 IEEE IECON. 22nd International Conference on Industrial Electronics, Control and Instrumentation. 1996. Taipei Taiwan. 
15. Whitcomb, L.L., A. Rizzi, and D.E. Koditschek, Comparative experiments with a new adaptive controller for robot arms. IEEE Transactions on Robotics and Automation, 1993. 9(1): p. 59-70.

16. Whitcomb, L., L.,, et al., Adaptive model based hybrid control of geometrically constrained robot arms. IEEE Transactions on Robotics and Automation, 1997. 13(1): p. 105-116.

17. Suzuki, H. and S. Arimoto, Visual control of autonomous mobile robot based on selforganizing model for pattern learning. Journal of Robotic Systems, 1988. 5(5): p. 453-470.

18. Krotkov, E. and R. Simmons. Performance of a six-legged planetary rover: power, positioning and autonomous walking. in Proc IEEE Int Cong Proc Robt Aut. 1992. Nice, France.

19. Yoerger, D.R., A.M. Bradley, and B.B. Walden, Autonomous benthic explorer deep ocean scientific auv for seafloor exploration: Untethered on station one year without support ship. Sea Technology, 1992: p. 50-54.

20. Xu, Y. and T. Kanade, Space robotics: Dynamics and control. 1993, Boston, MA USA: Kluwer.

21. Morikawa, H. and N. Takanashi. Ground experiment system for space robots based on predictive bilateral control. in IEEE Conf on Robotics and Automation. 1996. Minneapolis, MN USA: IEEE Press.

22. Guo, C., T.J. Tarn, and A. Bejczy. Fusion of human and machine intelligence for telerobotic systems. in IEEE Int Joint Conf on Robotics and Automation. 1995. Nagoya, JP: IEEE Press.

23. Kazerooni, H. Human/robot interaction via the transfer of power and information signals -- part $i$ : Dynamics and control analysis. in Proc IEEE Int Conf on Robotics and Automation. 1989.

24. Kazerooni, H. Human/robot interaction via the transfer of power and information signals -- part ii: Dynamics and control analysis. in Proc IEEE Int Conf on Robotics and Automation. 1989.

25. Kazerooni, H. and G. Jenhwa, Human extenders. Transaction of the ASME: Journal of Dynamic Systems, Measurement and Control, 1993. 115(2B): p. 218-90, June.

26. Cho, Y., T. Kotoku, and K. Tanie. Discrete-event-planning and control of telerobotic part mating process with communication delay in geomtric uncertainty. in IEEE Conf on Intelligent Robots \& Systems. 1995. Pittsburgh, PA USA: IEEE Press.

27. Yamamoto, Y., H. Eda, and X. Yun. Coordinated task execution of a human and a mobile manipulator. in IEEE Int Conf on Robotics and Automation. 1996. Minneapolis, MN USA: IEEE Press.

28. Bargar, W., et al. Robodoc Multi-Center Trial: An Interim Report. in Proc. 2nd Int. Symp. on Medical Robotics and Computer Assisted Surgery. 1995. Baltimore, Md.: MRCAS '95 Symposium, C/O Center for Orthop Res, Shadyside Hospital, Pittsburgh, Pa.

29. Taylor, R.H., et al., An Image-directed Robotic System for Precise Orthopaedic Surgery. IEEE Transactions on Robotics and Automation, 1994. 10(3): p. 261-275.

30. Mittelstadt, B.D., et al. The Evolution of a Surgical Robot from Prototype to Human Clinical Trial. in Proc. Medical Robotics and Computer Assisted Surgery. 1994. Pittsburgh.

31. Funda, J., et al. Image Guided Command and Control of a Surgical Robot. in Proc. Medicine Meets Virtual Reality II. 1994. San Diego.

32. Eldridge, B., et al., A Remote Center of Motion Robotic Arm for Computer Assisted Surgery. Robotica, 1996. 14(1 (Jan-Feb)): p. 103-109.

33. Funda, J., et al. Optimal Motion Control for Teleoperated Surgical Robots. in 1993 SPIE Intl. Symp. on Optical Tools for Manuf. \& adv. Autom. 1993. Boston.

34. Funda, J., et al. An experimental user interface for an interactive surgical robot. in 1st International Symposium on Medical Robotics and Computer Assisted Surgery. 1994. Pittsburgh. 
35. Funda, J., et al. Comparison of two mainpulator designs for laparoscopic surgery. in 1994 SPIE Int's Symposium on Optical Tools for Manufacturing and Advanced Automation. 1994. Boston: October.

36. Goradia, T.M., R.H. Taylor, and L.M. Auer. Robot-assisted minimally invasive neurosurgical procedures: first experimental experience. in Proc. First Joint Conference of CVRMed and MRCAS. 1997. Grenoble, France: Springer.

37. Taylor, R.H., et al., A Telerobotic Assistant for Laparoscopic Surgery, in IEEE EMBS Magazine Special Issue on Robotics in Surgery. 1995. p. 279-291.

38. Taylor, R.H., et al., A Telerobotic Assistant for Laparoscopic Surgery, in ComputerIntegrated Surgery, R. Taylor, et al., Editors. 1996, MIT Press. p. 581-592.

39. Harris, S.J., et al. Experiences with robotic systems for knee surgery. in Proc. First Joint Conference of CVRMed and MRCAS. 1997. Grenoble, France: Springer.

40. Ho, S.C., R.D. Hibberd, and B.L. Davies, Robot Assisted Knee Surgery. IEEE EMBS Magazine Sp. Issue on Robotics in Surgery, 1995(April-May): p. 292-300.

41. Troccaz, J., M. Peshkin, and B.L. Davies. The use of localizers, robots, and synergistic devices in CAS. in Proc. First Joint Conference of CVRMed and MRCAS. 1997. Grenoble, France: Springer.

42. Kumar, R., et al., Robot-assisted microneurosurgical procedures, comparative dexterity experiments, in Society for Minimally Invasive Therapy 9th Annual Meeting, Abstract book Vol 6, supplement 1. 1997: Tokyo, Jaban.

43. Kontarinis, D.A. and R.D. Howe, Tactile Display of Vibratory Information in Teleoperation and Virtual Environments. Presence, 1995. 4(4): p. 387-402.

44. Stoianovici, D., Cadeddu, J., A., Whitcomb, L., L., Taylor, R., H., Kavoussi, L., R.,. A Robotic System for Precise Percutaneous Needle Insertion,. in Thirteenth Annual Meeting of the Society for Urology and Engineering. 1988. San Diego.

45. Stoianovici, D., et al. A Modular Surgical Robotic System for Image-Guided Percutaneous Procedures. in Medical Image Computing and Computer-Assisted Interventions (MICCAI98). 1998. Cambridge, Mass: Springer.

46. Cadeddu, J.A., et al., A Robotic System for Percutaneous Renal Access Incorporating a Remote Center of Motion Design. Journal of Endourology, 1998. 12: p. S237.

47. Bishoff, J.T., et al., RCM-PAKY: Clinical Application of a New Robotic System for Precise Needle Placement. Journal of Endourology, 1998. 12: p. S82.

48. Kumar, R., et al. Performance of Robotic Augmentation in Microsurgery-Scale Motions. in 2nd Int. Symposium on Medical Image Computing and Computer-Assisted Surgery. 1999. Cambridge, England.

49. Humayun, M.U., et al., Quantitative measurement of the effects of caffiene and propranolol on surgeon hand tremor. Arch. Opthomol., 1997. 115: p. 371-374.

50. Riviere, C.N. and N.V. Thakor, Modeling and canceling tremor in human-machine interfaces. IEEE Eng. in Med. \& Biol. Magazine, 1996(May/June): p. 29-36.

51. Riviere, C.N. and P.K. Khosla. Microscale measurement of surgical instrument motion. in 2nd Intl. Conf on Medical Image Computing and Computer-Assisted Interventions (MICCAI 99). 1999. Cambridge, England.

52. Riviere, C.N. and P.K. Khosla. Intraoperative tremor monitoring for vitreoretinal microsurgery. in 2nd Intl. Conf on Medical Image Computing and Computer-Assisted Interventions (MICCAI 99). 1999. Cambridge, England.

53. Riviere, C.N. and P.K. Khosla. Augmenting the human-machine interface: improving manual accuracy. in Proceedings of the IEEE International Conference on Robotics and Automation. 1997. Albuquerque.

54. Jensen, P.S. and J. de Juan, E., In-vivo microscopy using gradient index of refraction (GRIN) lens endoscopy. Journal of Biomedical Optics, 1999((in review)). 